\title{
The accelerated scaling attractor solution of the interacting agegraphic dark energy in Brans-Dicke theory
}

\author{
Xian-Ming Liu 园12, Zhong-Xu Zhai2, Kui Xiao 目, Wen-Biao Liu \\ (corresponding author) \\ ${ }^{1}$ Department of Physics, Hubei University for Nationalities, Enshi Hubei, 445000, China \\ ${ }^{2}$ Department of Physics, Institute of Theoretical Physics, Beijing Normal University, Beijing, 100875, China \\ ${ }^{3}$ Department of Basic Teaching, Hunan Institute of Technology, Hengyang, 421002, China
}

Received: date / Accepted: date

\begin{abstract}
We investigate the interacting agegraphic dark energy in Brans-Dicke theory and introduce a new series general forms of dark sector coupling. As examples, we select three cases involving a linear interaction form (Model I) and two nonlinear interaction form (Model II and Model III). Our conclusions show that the accelerated scaling attractor solutions do exist in these models. We also find that these interacting agegraphic dark energy modes are consistent with the observational data. The difference in these models is that nonlinear interaction forms give more approached evolution to the standard $\Lambda \mathrm{CDM}$ model than the linear one. Our work implies that the nonlinear interaction forms should be payed more attention.
\end{abstract}

PACS 95.36.+x · 98.80.-k 9 98.80.Es

\section{Introduction}

Recent astronomical observations have provided strong evidence that our universe is undergoing an accelerated expansion due to an exotic energy component with negative pressure which is called dark energy [1,2,3,4, By far a leading candidate of dark energy model is the Einstein's cosmological constant model ( $\Lambda \mathrm{CDM})$ which is consistent with the late-time observational data. However, it is confronted with the so-called "cosmological constant problem" and "coincidence problem" [5]. Therefore, many dynamical dark energy models [6], such as quintessence [7, phantom 8, quintom 9], tachyon [10, generalized Chaplygin gas [11], etc, have also been taken into account.

The cosmological constant problem may be essentially an issue of quantum gravity problem, since the

\footnotetext{
a e-mail: xianmingliu@mail.bnu.edu.cn

be-mail: wbliu@bnu.edu.cn
}

cosmological constant is commonly considered as the vacuum expectation value of some quantum fields. Although a complete theory of quantum gravity has not been established, according to some principles of quantum gravity, one can make some attempts to probe the nature of dark energy model. The holographic dark energy model is just an appropriate and interesting example. This model is based on the holographic principle of quantum gravity theory [12, and is derived from the relationship between the ultraviolet (UV) and the infrared (IR) cutoffs proposed by Cohen et al. in ref. [13. According to the limit set of the formation of a black hole, the UV-IR relationship gives an upper bound on the zero point energy density $\rho_{q}=3 L^{-2} / 8 \pi G$, which means that the maximum entropy of the system is of the order of $S_{B H}^{3 / 4}$, where $L$ is the scale of IR cutoff and $S_{B H}$ is the entropy of the black hole. Compared with the holographic dark energy model and based on the Károlyházy relation [14, the so-called agegraphic dark energy model was proposed, where the age of the universe $T=\int d t$ is used as the IR cutoff $L[15$. Furthermore, in ref. 16, the interacting agegraphic dark energy has been introduced and investigated. It was shown that the equation of state of interacting agegraphic dark energy can cross the phantom division. The interacting agegraphic dark energy model also has been extended to the universe with spatial curvature in ref. [17. Recently, using the phase space analysis, it was shown that the accelerated scaling attractor solutions of the interacting agegraphic dark energy in the Einstein universe did exist and the results agree with the observations [18.

On the other side, scalar-tensor theories of gravity have been widely applied to cosmology 19. The simplest alternative to Einstein's general relativity which includes a scalar field in addition to the tensor field 
is Brans-Dicke theory. This theory is more consistent with the Mach's principle and less reliant on absolute properties of space [20]. It got a new impetus in recent years because it arises naturally as the low energy limit of many theories of quantum gravity such as super string theory or Kaluza-Klein theory. Noticing that the holographic dark energy density belongs to a dynamical cosmology constant, it is more natural for a dynamical frame to replace the Einstein's general gravity. Therefore, it is worthwhile to investigate the holographic dark energy model within the framework of Brans-Dicke theory $[21,22,23,24]$. The extended holographic dark energy model with Hubble horizon in Brans-Dicke theory has been proposed and it is found that the model is not a viable dark energy model unless the Brans-Dicke scalar field has a potential. So it is a very interesting attempt to deeply investigate the agegraphic dark energy model in the framework of Brans-Dicke theory .

Considering that dark energy (DE) and dark matter (DM) contribute to the most fraction of the content of the universe, it is natural to look into the possibility of the interaction between DE and DM, which has been widely discussed [26]. It has been argued that the coupling between DE and DM can provide a mechanism to alleviate the coincidence problem and lead to an accelerated scaling attractor solution with similar energy densities in the dark sector today [26,27, 28, 29]. Noticing that there is no fundamental theory which can be used to select a specific interacting dark energy model, any interacting dark energy model will necessarily be phenomenological. There are two criterions to determine whether the model is correct and feasible. One is the observations, the other is to examine whether the interacting model can lead to the accelerated scaling attractor solutions, which is a decisive way to achieve similar energy densities in dark sector and alleviate the coincidence problem. In this work, we firstly introduce a new series of interacting agegraphic dark energy models including linear and nonlinear forms. Using the phaseplane analysis, it is found that the accelerated scaling attractor solutions do exist in these models. What's more, these agegraphic dark energy models are in accordance with the late-time observational data.

Our paper is organized as follows. In section 2, the agegraphic dark energy models in Brans-Dicke theory is constructed and a series of dark sector coupling forms are introduced. In section 3 using the phase-plane analysis, the accelerated scaling attractor solutions are discussed in these models. In section 4, using the newly released Hubble parameter data $30,31,32,33$, these agegraphic dark energy models are tested. Some conclusions will be presented in section 5 .

\section{Construction of the interacting agegraphic dark energy in Brans-Dicke theory}

According to our metric convention, $(+,-,-,-)$, the Lagrangian for Brans-Dicke theory with a scalar field in the Jordan frame is

$\mathcal{L}_{B D}=\sqrt{-g}\left[\left(-\varphi R+\omega \frac{1}{\varphi} g^{\mu \nu} \partial_{\mu} \varphi \partial_{\nu} \varphi\right)+\mathcal{L}_{M}(\Psi)\right]$,

where the dimensionless $\omega$ is the coupling constant, and $\mathcal{L}_{M}(\Psi)$ is the matter Lagrangian. The current observational constraint on $\omega$ is $\omega>10^{4}$; it recovers Einstein's general relativity when $\omega \rightarrow \infty$. In particular, it is expected that $\varphi(t, \vec{x})$ is spatially uniform and evolves slowly only with cosmic time $t$ so that $\varphi(t, \vec{x}) \rightarrow \varphi(t)$. Following ref. [35, we can introduce a new field $\phi$ as

$\varphi=\frac{1}{8 \omega} \phi^{2}$.

So the Lagrangian for Brans-Dicke theory can be written as following form

$\mathcal{L}_{B D}=\sqrt{-g}\left[-\frac{1}{8 \omega} \phi^{2} R+\frac{1}{2} g^{\mu \nu} \partial_{\mu} \phi \partial_{\nu} \phi+\mathcal{L}_{M}(\Psi)\right]$.

Considering a classical perfect fluid with the energymomentum tensor $T_{\nu}^{\mu}=\operatorname{diag}(\rho,-p,-p,-p)$, the gravitational field equations derived from the variation of the action Eq. (3) with respect to the flat RobertsonWalker metric are

$$
\begin{aligned}
& \frac{3}{4 \omega} \phi^{2} H^{2}-\frac{1}{2} \dot{\phi}^{2}+\frac{3}{2 \omega} H \dot{\phi} \phi=\rho \\
& -\frac{1}{4 \omega} \phi^{2}\left(2 \dot{H}+3 H^{2}\right)-\frac{1}{\omega} H \dot{\phi} \phi-\left(\frac{1}{2}+\frac{1}{2 \omega}\right) \dot{\phi}^{2}-\frac{1}{2 \omega} \ddot{\phi} \phi=(5) \\
& \ddot{\phi}+3 H \dot{\phi}=\frac{3}{2 \omega}\left(\dot{H}+2 H^{2}\right) \phi \\
& \dot{\rho}+3 H(\rho+p)=0,
\end{aligned}
$$

where the dot is the derivative with respect to time and $H=\frac{\dot{a}}{a}$ is the Hubble parameter. Combining the above equations, we get

$-\dot{H}=-4\left(\frac{H \dot{\phi}}{\phi}\right)+2 \omega\left(\frac{\dot{\phi}}{\phi}\right)^{2}+\frac{4 \omega}{(2 \omega+3) \phi^{2}}[\omega p+(\omega+2) \rho]$.

The total matter is supposed to be composed of two parts: pressureless dark matter and agegraphic dark energy. So the total energy density $\rho$ includes the energy density of agegraphic dark energy $\rho_{q}$ and the energy density of dark matter $\rho_{m}$ in this model. In Brans-Dicke theory, the effective gravitational constant $G_{\text {eff }}$ can be defined as $G_{\text {eff }}^{-1}=\frac{2 \pi}{\omega} \phi^{2}$, the agegraphic dark energy can naturally be defined as

$\rho_{q}=\frac{3 n^{2} \phi^{2}}{4 \omega T^{2}}$ 
where $n$ is a positive constant. Now we turn to considering the interaction between dark matter and dark energy. The balance equations of the agegraphic dark energy and dark matter can be written respectively as 23

$$
\begin{aligned}
& \dot{\rho}_{q}+3 H\left(1+\omega_{q}\right) \rho_{q}=-Q, \\
& \dot{\rho}_{m}+3 H \rho_{m}=Q,
\end{aligned}
$$

where $Q$ denotes the phenomenological interaction term. The interacting term is always a function of the Hubble parameter $H$, the density of dark energy, and the density of the dark matter. One can often find the following interaction model forms [18, 16, 36, 26, 37, 38, 39, 40, 23.

$$
\begin{aligned}
Q= & 3 H \alpha \rho_{q}, 3 H \beta \rho_{m}, 3 \gamma H\left(\rho_{q}+\rho_{m}\right), \\
& 3 H\left(\alpha \rho_{q}+\beta \rho_{m}\right), 3 \beta H \rho_{q}^{\alpha} \rho_{m}^{1-\alpha},
\end{aligned}
$$

where $\alpha, \beta, \gamma$ are positive constant parameters. We would like to consider the more general interaction as

$Q=3 H \rho_{q} g(\xi)=3 H \rho_{q} \sum_{i=-\infty}^{+\infty} A_{i} \xi^{i}$

where $\xi=\frac{\rho_{m}}{\rho_{q}}, g(\xi)=\sum_{i=-\infty}^{+\infty} A_{i} \xi^{i}$, and $A_{i}$ is a positive constant parameter with respect to $\xi^{i}$. Then Eq.(10) and Eq.111) can be written as

$$
\begin{aligned}
& \dot{\rho}_{q}+3 H\left(1+\omega_{q}\right) \rho_{q}=-3 H \rho_{q} g(\xi), \\
& \dot{\rho}_{m}+3 H \rho_{m}=3 H \rho_{q} g(\xi) .
\end{aligned}
$$

In this paper, as examples, $g(\xi)$ will be selected as:

$$
\begin{aligned}
\text { Model I: } & g_{1}(\xi)=\alpha_{1}+\beta_{1} \xi \\
\text { Model II: } & g_{2}(\xi)=\alpha_{2}+\beta_{2} \xi^{2}, \\
\text { Model III: } & g_{3}(\xi)=\alpha_{3} \xi^{-1}+\beta_{3} \xi
\end{aligned}
$$

It is obvious that Model I is linear interaction, while Model II and III are nonlinear interactions, which can be taken as the extending of Eq. (12).

\section{Phase-space analysis}

According to the discussion in section 2 it is easy to see that eqs. (4), (6), (8), (14), and (15) can give a closed system which can determine the cosmic behavior. In order to study the dynamical behavior of interacting dark energy and dark matter, we further introduce the following dimensionless variables

$x=\sqrt{\frac{4 \omega}{3 \phi^{2} H^{2}} \rho_{q}}, y=\sqrt{\frac{4 \omega}{3 \phi^{2} H^{2}} \rho_{m}}, \lambda=\frac{\dot{\phi}}{H \phi}$.

Similar forms of selecting these dimensionless variables in agegraphic dark energy model was firstly used in ref.
18. Using these dimensionless variables, Eq. (44) becomes

$x^{2}+y^{2}+\frac{2 \omega}{3} \lambda^{2}-2 \lambda=1$.

Now, the critical density can be defined as $\rho_{c}=\frac{3 H^{2}}{8 \pi G_{\text {eff }}}=$ $\frac{3 \phi^{2} H^{2}}{4 \omega}$ 24], so Eq. (20) becomes $\Omega_{q}+\Omega_{m}+\Omega_{\phi}=1$, where

$\Omega_{q}=x^{2}, \Omega_{m}=y^{2}, \Omega_{\phi}=\frac{2 \omega}{3} \lambda^{2}-2 \lambda$.

From Eq. (21), we find that the Brans-Dicke scalar field $\phi$ plays a role of dark energy, so we can assume that both the agegraphic and the scalar field drive our universe to accelerate [24]. So we have

$\Omega_{D E}=\Omega_{q}+\Omega_{\phi}$,

then

$r=\frac{\Omega_{D M}}{\Omega_{D E}}=\frac{\Omega_{m}}{\Omega_{q}+\Omega_{\phi}}=\frac{1}{x^{2}+\frac{2 \omega}{3} \lambda^{2}-2 \lambda}-1$.

Noticing that $0 \leq \Omega_{q}, \Omega_{\phi}, \Omega_{m} \leq 1$, the limit of $x, y$, and $\lambda$ can be obtained as $0 \leq x \leq 1,0 \leq y \leq 1$, $\frac{3}{2 \omega}\left(1-\sqrt{1+\frac{2 \omega}{3}}\right) \leq \lambda \leq 0$ or $\frac{3}{\omega} \leq \lambda \leq \frac{3}{2 \omega}\left(1-\sqrt{1+\frac{2 \omega}{3}}\right)$ respectively.

Subsequently, thinking of Eqs. (4)- (8), we have

$$
\begin{aligned}
x^{\prime} & =-x\left[f(x, y, \lambda)+\frac{x}{n}\right], \\
y^{\prime} & =-y\left[\frac{3}{2}\left(1-\frac{x^{2} g(\xi)}{y^{2}}\right)+\lambda+f(x, y, \lambda)\right], \\
\lambda^{\prime} & =-3 \lambda-\lambda^{2}+\frac{3}{\omega}+\left(\frac{3}{2 \omega}-\lambda\right) f(x, y, \lambda),
\end{aligned}
$$

where $x^{\prime}=d x / d N, y^{\prime}=d y / d N, \lambda^{\prime}=d \lambda / d N, N=$ $\ln a, \xi=\frac{y^{2}}{x^{2}}$, and

$$
\begin{aligned}
f(x, y, \lambda) & =\frac{\dot{H}}{H^{2}}=4 \lambda-2 \omega \lambda^{2}-\frac{3 \omega}{2 \omega+3}\left[1-g(\xi)-\frac{2}{3} \lambda+\frac{2}{3 c} x\right] x^{2} \\
& -\frac{3(\omega+2)}{2 \omega+3}\left(1-\frac{2 \omega}{3} \lambda^{2}+2 \lambda\right) .
\end{aligned}
$$

The state parameter $\omega_{q}$ for the agegraphic dark energy could be expressed in terms of these new variables as

$\omega_{q}=-1+\frac{2}{3 n} x-\frac{2}{3} \lambda-g(\xi)$.

For completeness, we give the deceleration parameter

$q=-\frac{\ddot{a}}{a H^{2}}=-1-\frac{\dot{H}}{H^{2}}=-1-f(x, y, \lambda)$.

In the interacting agegraphic dark energy models, the properties of agegraphic dark energy are determined by the parameters $A_{i}, \omega$ and $n$. Eqs. (24)-(27) are just the functions of $x, y$, and $\lambda$, not the functions of $N$ or 
other variables. So this dynamical system is just a threedimensional autonomous system. For an autonomous system $\mathbf{X}^{\prime}=f(\mathbf{X})$, there are some critical points $\mathbf{X}_{c}$ satisfying $\mathbf{X}^{\prime}=0$, so we have

$$
\begin{aligned}
& -x_{c}\left(f\left(x_{c}, y_{c}, \lambda_{c}\right)+\frac{x_{c}}{n}\right)=0, \\
& -y_{c}\left[\frac{3}{2}\left(1-\frac{x_{c}^{2} g\left(\xi_{c}\right)}{y_{c}^{2}}\right)+\lambda_{c}+f\left(x_{c}, y_{c}, \lambda_{c}\right)\right]=0, \\
& -3 \lambda_{c}-\lambda_{c}^{2}+\frac{3}{\omega}+\left(\frac{3}{2 \omega}-\lambda_{c}\right) f\left(x_{c}, y_{c}, \lambda_{c}\right)=0,
\end{aligned}
$$

where $\xi_{c}=\frac{y_{c}^{2}}{x^{2}}$. In order to determine the stability property of the critical points, it is necessary to expand the autonomous system $\mathbf{X}^{\prime}=f(\mathbf{X})$ around the critical points $\mathbf{X}_{c}$. Setting $\mathbf{X}=\mathbf{X}_{c}+\mathbf{U}$, where $\mathbf{U}$ is a column vector of the perturbation of the variables, one can expand the equation for the perturbation up to the first order as $\mathbf{U}^{\prime}=\mathbf{M} \cdot \mathbf{U}$, where the matrix $\mathbf{M}$ contains the coefficients of the perturbation equations. The stability property for each critical point is determined by the eigenvalues of $\mathbf{M} 25$. The $3 \times 3$ matrix $\mathbf{M}$ of the linearized perturbation equations is

$$
\begin{gathered}
\mathbf{M}=\left(\begin{array}{c}
-x\left(\frac{\partial f}{\partial x}+\frac{2}{n}\right) \\
-y\left(-\frac{3 x}{y^{2}} g(\xi)+\frac{3}{x} \frac{\partial g(\xi)}{\partial \xi}+\frac{\partial f}{\partial x}\right) \\
\left(\frac{3}{2 \omega}-\lambda\right) \frac{\partial f}{\partial x} \\
-x \frac{\partial f}{\partial y} \\
-\left[\frac{3}{2}+\lambda+f+\frac{3 x^{2}}{2 y^{2}} g(\xi)-3 \frac{\partial g(\xi)}{\partial \xi}+y \frac{\partial f}{\partial y}\right] \\
\left(\frac{3}{2 \omega}-\lambda\right) \frac{\partial f}{\partial y} \\
-x \frac{\partial f}{\partial \lambda} \\
-y\left(1+\frac{\partial f}{\partial \lambda}\right) \\
-3-2 \lambda-f+\left(\frac{3}{2 \omega}-\lambda\right) \frac{\partial f}{\partial \lambda}
\end{array}\right),
\end{gathered}
$$

where

$$
\begin{aligned}
& \frac{\partial f}{\partial x}=-\frac{6 \omega}{2 \omega+3}\left[\frac{x^{2}}{n}-x g(\xi)+\frac{y^{2}}{x} \frac{\partial g(\xi)}{\partial \xi}\right], \\
& \frac{\partial f}{\partial y}=y \frac{6 \omega}{2 \omega+3} \frac{\partial g(\xi)}{\partial \xi}, \\
& \frac{\partial f}{\partial \lambda}=4(1-\omega \lambda)-\frac{6(\omega+2)}{2 \omega+3}\left(1-\frac{2}{3} \omega \lambda\right)+\frac{2 \omega}{2 \omega+3} x^{2}, \\
& \frac{\partial g(\xi)}{\partial \xi}=\sum_{i=-\infty}^{+\infty} i A_{i} \xi^{i-1} .
\end{aligned}
$$

We can examine the sign of the real part of the eigenvalues of $\mathbf{M}_{x=x_{c}, y=y_{c}, \lambda=\lambda_{c}}$, which determines the type and stability of the critical points $\left(x_{c}, y_{c}, \lambda_{c}\right)$. The simplest finite critical points and their properties for this model are summarized in the Table 1] Here we only concentrate on the attractor, so the real part of the eigenvalues of $\mathbf{M}_{x=x_{*}, y=y_{*}, \lambda=\lambda_{*}}$ should be negative. If the autonomous system of Eqs.(24), (25) and (26) presents scaling solutions, the coincidence problem gets substantially alleviated because, regardless of the initial conditions, the system evolves toward a final state where the

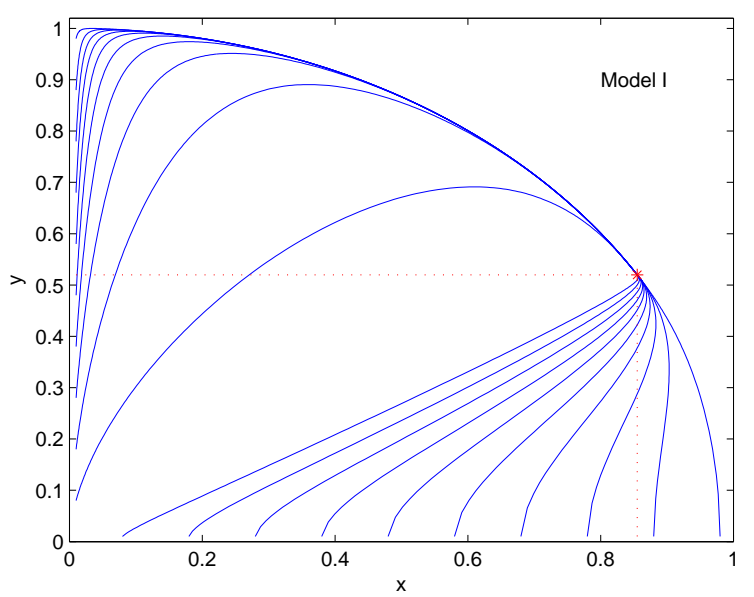

Fig. 1 The phase plane for the model I with $n=20, \omega=10^{3}$, $\alpha_{1}=0.3$ and $\beta_{1}=0.16$. The red star stands for the late-time attractor with $x_{*} \approx 0.86, y_{*} \approx 0.26, \lambda_{*} \approx 9.9 \times 10^{-4}$.

ratio of dark matter to dark enenrgy stays constant. Using Eq. (23), it is easy to find that

$r^{\prime}=-\left(1+r^{2}\right)\left[2 x x^{\prime}+\left(\frac{4 \omega}{3} \lambda-2\right) \lambda^{\prime}\right]$

The scaling solutions mean $r^{\prime}=0$. Obviously the attractor $\left(x_{*}, y_{*}, \lambda_{*}\right)$ is just a scaling solution, because $\left.x^{\prime}\right|_{x_{*}}=0,\left.\lambda^{\prime}\right|_{\lambda_{*}}=0$ lead to $\left.r^{\prime}\right|_{x_{*}, y_{*}, \lambda_{*}}=0$. What's more, if the critical point is the accelerated scaling attractor solution, the following constraints $q\left(x_{*}, y_{*}, \lambda_{*}\right)<$ $0, r\left(x_{*}, y_{*}, \lambda_{*}\right) \approx \frac{0.26}{0.74} \approx 0.353$ should also be satisfied.

Obviously the accelerated scaling attractor solutions can be obtained by fixing the parameters $A_{i}, \omega$, and $n$. We have found the accelerated scaling attractor solutions in Model I, Model II, and Model III using the numerical analysis method. The results are shown in Fig. 1, 2, 3. respectively. The numerical solutions show that the cosmic evolution is insensitive to the initial conditions. The different interacting model forms just affect the intermediate evolution. This makes the coincidence problem to be substantially alleviated.

Figures 4, 5, 6, show that the three interacting agegraphic dark energy models give very similar conclusions. From Fig. 4, one can see the cosmic evolution in these models, where $\Omega_{\phi}$ dominates the universe at the early epoch and then the matter is becoming increasingly dominant over $\Omega_{\phi}$ afterwards. After a transitory matter-dominated epoch, the universe becomes accelerated expansion in the agegraphic dark energy dominated epoch. From Fig. 5, the universe is now undergoing an accelerated expansion phase. The similar curves of $q$ in three models show that this cosmic acceleration is arising recently. The evolution of equations of state of dark energy is presented in Fig. 6. There is a phantom- 
Table 1 Location of the critical points of the autonomous system of Eqs. (24), 25) and (26), their stability and dynamical behavior of the Universe at those points.

\begin{tabular}{lcc}
\hline$\left(x_{c}, y_{c}, \lambda_{c}\right)$ coordinates & Stability character & $\mathrm{q}$ \\
\hline$\left(0,0, \lambda_{1}=\frac{3}{2 \omega}\left(1 \pm \sqrt{1+\frac{2 \omega}{3}}\right)\right)$ & unstable & $-4-4 \lambda_{1}$ \\
$\left(0, y_{2}=\sqrt{1-\frac{2 \omega}{3} \lambda_{2}^{2}+2 \lambda_{2}}, \lambda_{2}=\frac{3(\omega+1)}{8 \omega}\left[1 \pm \sqrt{1+\frac{56 \omega}{3(\omega+1)^{2}}}\right]\right)$ & unstable & $-1-\frac{6 \omega \lambda_{2}+\omega \lambda_{2}-6}{3-2 \omega \lambda_{2}}$ \\
$\left(x_{3}, 0, \lambda_{3}=\frac{3}{2 \omega}\left[1 \pm \sqrt{1+\frac{2 \omega}{3}\left(1-x_{3}^{2}\right)}\right]\right)$ & unstable & $-1+\frac{x_{3}}{n}$ \\
$\left(x_{*}=\frac{n\left(6 \omega \lambda_{2}^{2}+\omega \lambda_{2}-6\right)}{3-2 \omega \lambda_{2}}, y_{*}=\sqrt{1-\frac{2 \omega}{3} \lambda_{*}^{2}+2 \lambda_{*}}-x_{*}^{2}, \lambda_{*}\right)$ & attractor & $-1+\frac{x_{*}}{n}$ \\
\hline
\end{tabular}

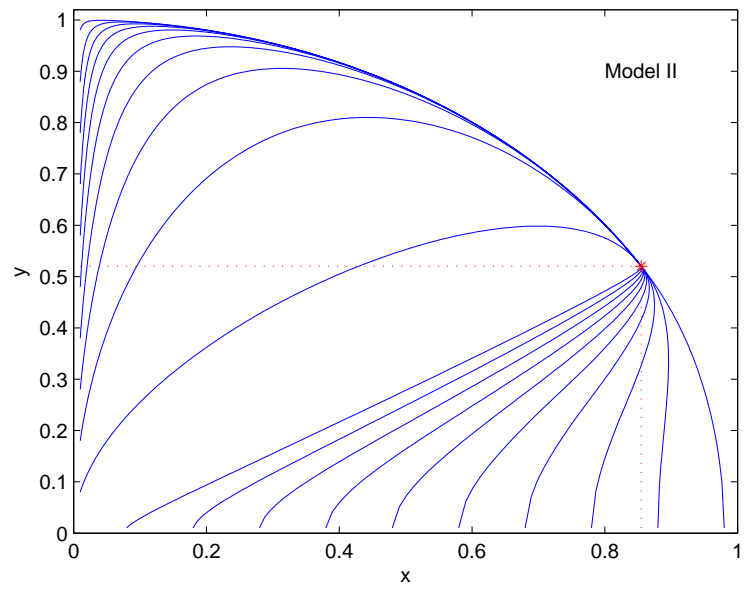

Fig. 2 The phase plane for the model II with $n=20, \omega=$ $10^{3}, \alpha_{2}=0.36$ and $\beta_{2}=10^{-6}$. The red star stands for the late-time attractor with $x_{*} \approx 0.86, y_{*} \approx 0.26, \lambda_{*} \approx 9.9 \times$ $10^{-4}$.

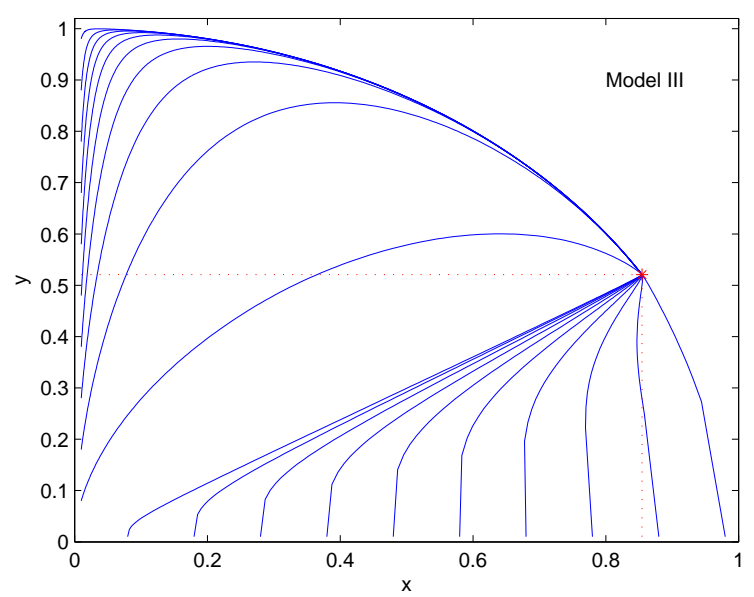

Fig. 3 The phase plane for the model III with $n=20, \omega=$ $10^{3}, \alpha_{3}=0.12$ and $\beta_{3}=0.1$. The red star stands for the latetime attractor with $x_{*} \approx 0.86, y_{*} \approx 0.26, \lambda_{*} \approx 9.9 \times 10^{-4}$. like to quintessence-like transition while the values of $\omega_{q}^{\prime}$ is always around -1 .

\section{Observational data}

Now, we would like to check the three interacting agegraphic dark energy models using the observational data and compare them with the standard $\Lambda$ CDM model.

The continuity eqs. (14) and (15) can also be written in the standard forms

$$
\begin{aligned}
& \dot{\rho}_{q}+3 H\left(1+\omega_{q}^{\prime}\right) \rho_{q}=0, \\
& \dot{\rho}_{m}+3 H\left(1+\omega_{m}^{\prime}\right) \rho_{m}=0,
\end{aligned}
$$

where $\omega_{q}^{\prime}=-1-\frac{2}{3}\left(\lambda-\frac{x}{n}\right), \omega_{m}^{\prime}=\frac{g(\xi)}{\xi}$.

The Brans-Dicke field can be assumed as a power law of the scale factor 23

$\phi=\phi_{0} a^{\lambda}$

Noticing that the scalar field $\Omega_{\phi}$ in figure 4 is very infinitesimal in the late-time universe, we can assume that $\lambda$ is a constant $\lambda \approx \lambda_{c}$, and the product $\lambda_{c} \omega$ results order unity, which is consistent with refs. 23, 41 .

Substituting Eqs. (34), (35), and (36) into Eq. (44), one can get the evolution of Hubble parameter $H=$ $H_{0} E(z)$, where the expansion rate $E(z)$ can be expressed as

$$
\begin{aligned}
& E^{2}(z)=\left(1+2 \lambda_{c}-\frac{2 \omega}{3 \lambda_{c}^{2}}\right)^{-1}(1+z)^{2 \lambda_{c}} \\
& {\left[\Omega_{m 0} \exp \left(3 \int_{0}^{z} \frac{1+\omega_{m}^{\prime}}{1+z^{\prime}} d z^{\prime}\right)+\Omega_{q 0} \exp \left(3 \int_{0}^{z} \frac{1+\omega_{q}^{\prime}}{1+z^{\prime}} d z^{\prime}\right)\right]}
\end{aligned}
$$

where the subscript " 0 " denotes the present value.

We will use the newly released Hubble parameter data $30,31,32,33,34$ which is directly related to the expansion history of the universe by its definition: $H=$ $\dot{a} / a$ to determine a preferable interacting model. In the meantime, we choose the standard $\Lambda$ CDM model as the fiducial model which fits the observations best. The results are presented in Fig. 7. 

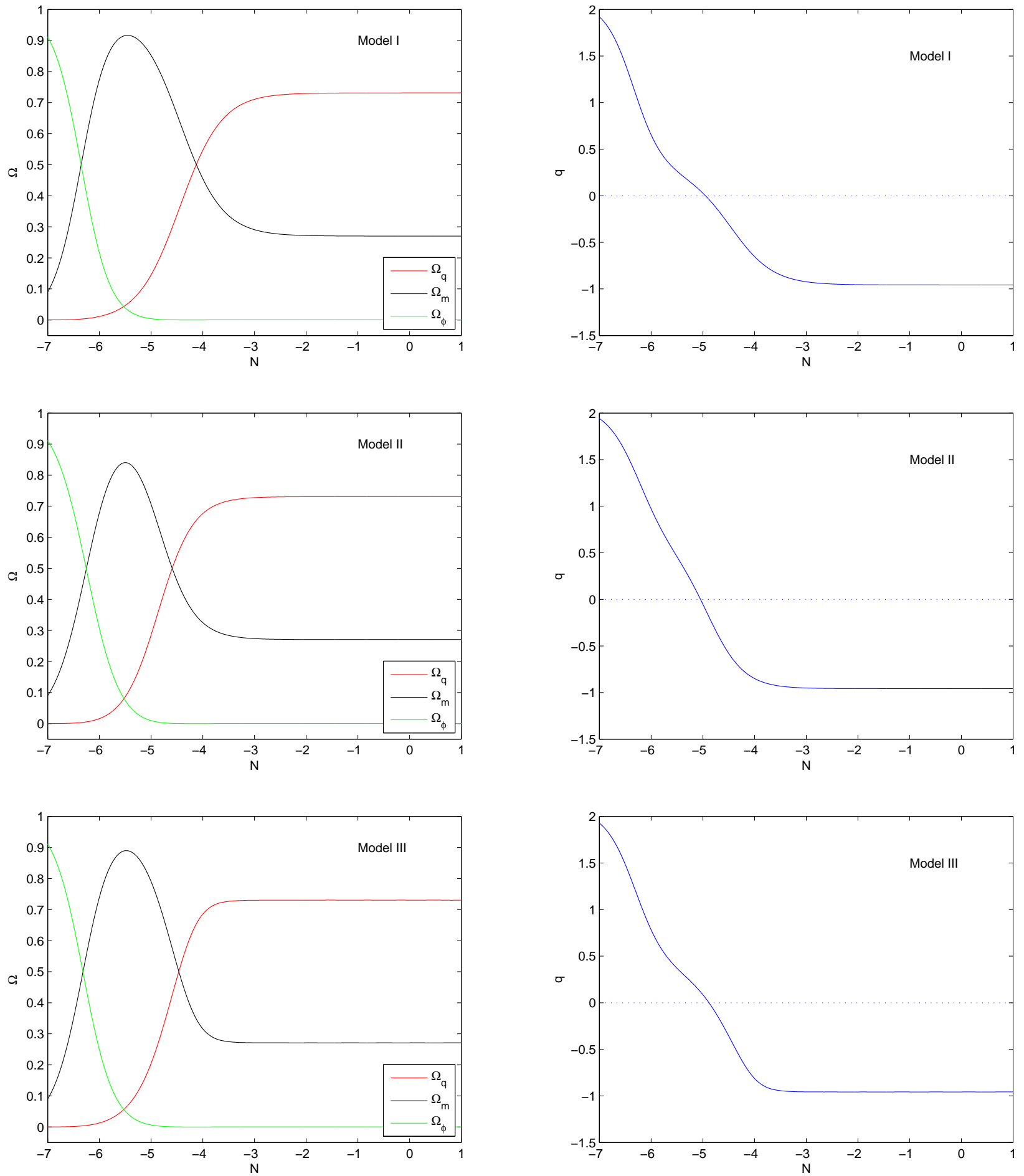

Fig. 4 Behavior of $\Omega_{m}$ (black), $\Omega_{q}$ (red) and $\Omega_{\phi}$ (green) as a function of $N=\ln a$ for the particular models

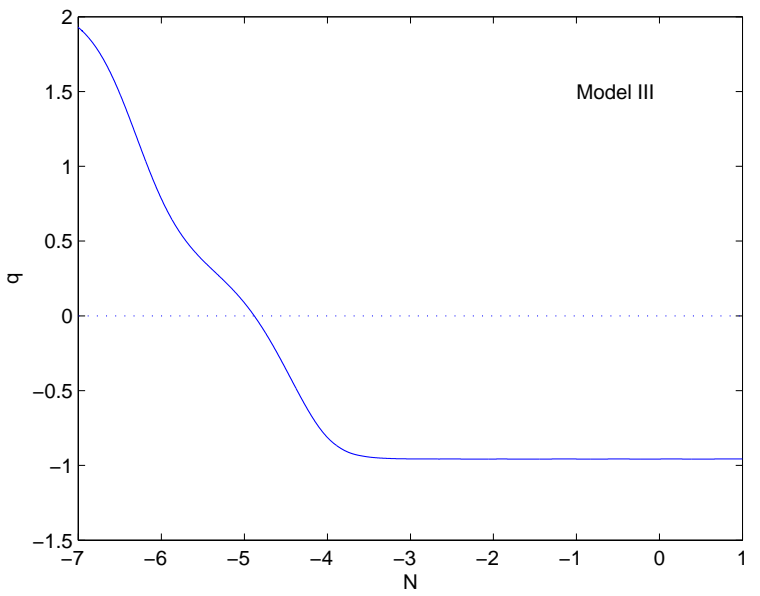

Fig. 5 Behavior of deceleration parameter $q$ as a function of $N=\ln a$ for the particular models 

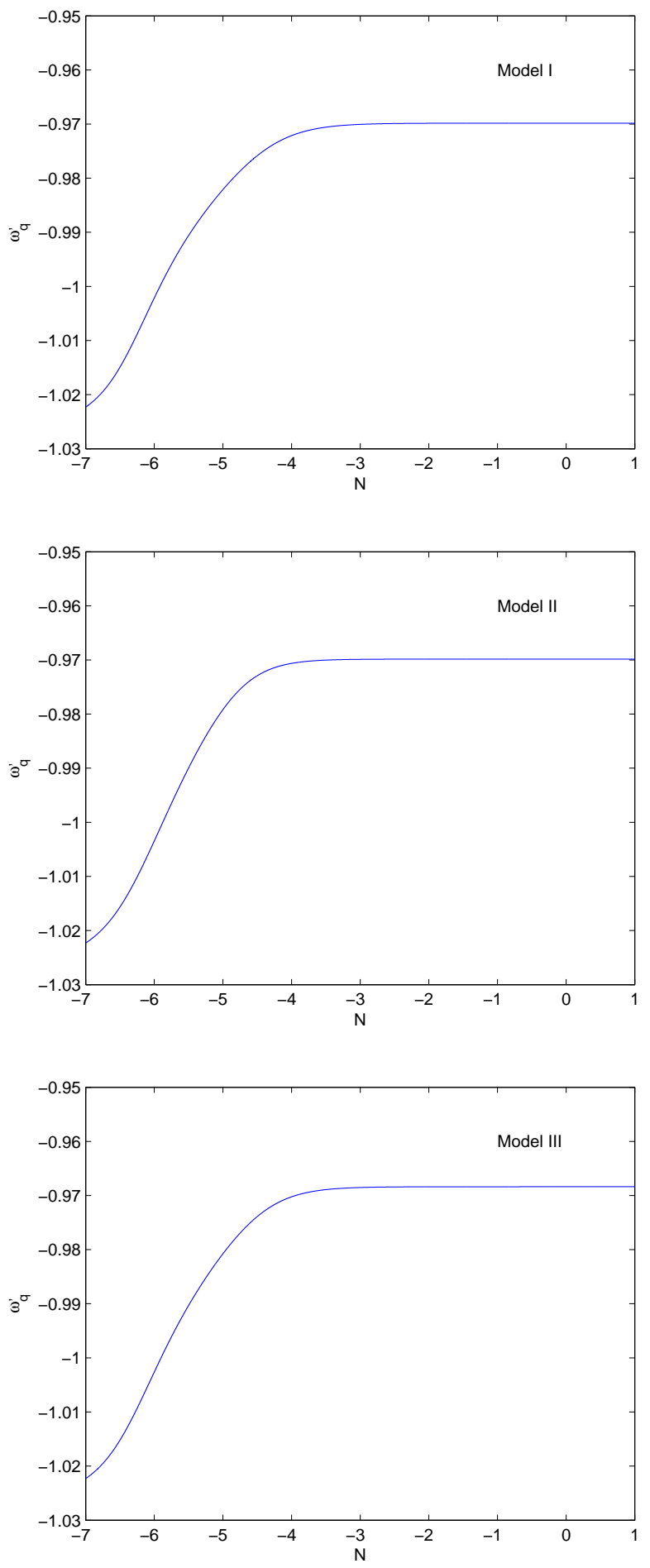

Fig. 6 Behavior of effective equation of state of dark energy $\omega_{q}^{\prime}$ as a function of $N=\ln a$ for the particular models

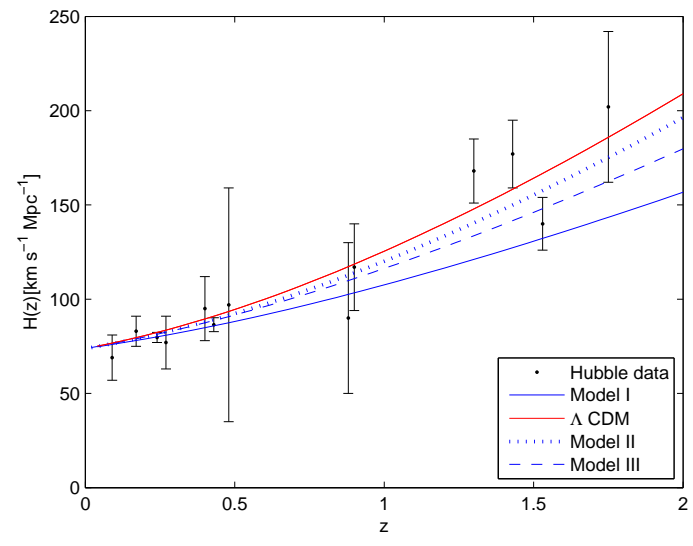

Fig. 7 The theoretical evolution of $H(z)$ with observational data points (black): $\Lambda \mathrm{CDM}$ (red solid), Model I (blue solid), Model II (blue dotted), and Model III (blue dashed)

Apparently, the nonlinear interaction forms (Model II and Model III) give better evolutions to the standard $\Lambda \mathrm{CDM}$ one. The linear one shows evident deviation from the $\Lambda \mathrm{CDM}$ and observation data. The nonlinear interaction between dark energy and dark matter is better to describe the real physical process. This comparison is incomplete and it is only a rough overview. It is necessary to further explore the nonlinear interaction between the dark section of the universe.

\section{Discussions and conclusions}

Holographic dark energy model is an interesting attempt to investigate the nature of dark energy in the framework of quantum gravity. Considering that the simplest alternative to Einstein's general relativity which includes a scalar field in addition to the tensor field is Brans-Dicke theory and the holographic bound can be satisfied for both the $k=0$ and $k=-1$ universe in the Brans-Dicke cosmology 42, it is natural to extend the research to the holographic dark energy models of this theory. In this paper, we have investigated the interacting agegraphic dark energy in the flat $(k=0)$ Brans-Dicke cosmology. Firstly, a series of new general forms of dark sector coupling are introduced and the accelerated scaling attractor solutions have been found. Moreover, using the newly released Hubble parameter data, we have also tested these interacting agegraphic dark energy models.

The interacting term can be selected as a function of $H$, the density of dark energy, and the density of the dark matter. According to this requirement, we have proposed the general interacting agegraphic dark energy models. Three cases including a linear interaction form (Model I) and two nonlinear interaction forms 
(Model II and Model III) have been investigated. Using the phase-plane analysis, the dynamical behavior of these models has been investigated and it was found that the accelerated scaling attractor solutions did exist in these models. This can alleviate the coincidence problem.

Afterwards, using the newly released Hubble parameter data, we tested these dynamical dark energy models. These interacting agegraphic dark energy modes have given a series of reasonable pictures of the cosmic evolution and they were consistent with the latetime observational data. In particular, we found the nonlinear interaction forms (Model II and Model III) gave more approached evolution to the standard $\Lambda$ CDM model than the linear one (Model I). Our work show that we should pay more attention to the nonlinear interaction forms rather than the linear form. This deserves further investigations.

Acknowledgements The authors would like to thank the anonymous referee for his/her insightful and constructive suggestions, which allowed us to improve the manuscript significantly. This work is supported by the National Natural Science Foundation of China under Grant Nos. 10773002, 10875012, and 11175019. It is also supported by the Fundamental Research Funds for the Central Universities under Grant No. 105116.

\section{References}

1. S. Perlmutter et al., Astrophys. J. 517565 (1999)[arXiv: astro-ph/9812133

2. C. L. Bennett et al., Astrophys. J. suppl. 1481 (2003) [arXiv: astro-ph/0302207]

3. M. Tegmark et al., Phys. Rev. D 69103501 (2004) [arXiv: astro-ph/0310723

4. S. W. Allen, R. W. Schmidt, H. Ebeling, A. C. Fabian and L. vani Speybroeck, Mon. Not. Roy. Astron. Soc. 353 457 (2004) [arXiv: astro-ph/0405340

5. S.Weinberg, Rev. Mod. Phys. 611 (1989);

S. Weinberg, The cosmological constant problems (Talk given at dark matter 2000, February, 2000), [arXiv: astro-ph/0005265.

6. E. J. Copland, M. Sami, S. Tsujikawa, Int. J. Mod. Phys. D 151753 (2006) [arXiv: hep-th/0603057.

7. B. Ratra and P. J. E. Peebles, Phys. Rev. D 373406 (1988);

C. Wetterich, Nucl. Phys. B 302 668(1988) ;

A. R. Liddle and R. J. Scherrer, Phys. Rev. D 59023509 (1999)[arXiv: astro-ph/9809272 ;

I. Zlatev, L. Wang and P. J. Steinhardt, , Phys. Rev. Lett. 82896 (1999) [arXiv: astro-ph/9807002];

Z. -K. Guo, N. Ohta and Y. -Z. Zhang, Mod. Phys. Lett.

A 22883 (2007)[arXiv: astro-ph/0603109] [SPIRES];

S. Dutta, E. N. Saridakis and R. J. Scherrer, Phys. Rev.

D 79103005 (2009)[arXiv: 0903.3412]

8. R. R. Caldwell, Phys. Lett. B 54523 (2002) [arXiv: astro-ph/9908168;

R. R. Caldwell, M. Kamionkowski and N. N. Weinberg, Phys. Rev. Lett. 91071301 (2003)[arXiv: astro-ph/0302506;
J. G. Hao, X. L. Li, Phys. Rev. D 68043501 (2003);

D. J. Liu, X. Z. Li, Phys. Rev. D 68 067301(2003);

S. Nojiri and S. D. Odintsov, Phys. Lett. B 562147 (2003)[arXiv: hep-th/0303117];

V. K. Onemli and R. P. Woodard, Phys. Rev. D 70 107301 (2004)[arXiv: gr-qc/0406098];

X. -M. Chen, Y. -G. Gong and E. N. Saridakis, JCAP 04 001 (2009) arXiv:0812.1117;

S. Dutta and R. J. Scherrer, Phys. Lett. B 67612 (2009) arXiv:0902.1004.

9. B. Feng, X. L. Wang, X. M. Zhang, Phys. Lett. B 607 35 (2005);

Y. -F. Cai, E. N. Saridakis, M. R. Setare, J. -Q. Xia, Phys. Rep. 4931 (2010).

10. X. Z. Li, J. G. Hao, D. J. Liu,Chin. Phys. Lett. 191584 (2002);

J. S. Bagla, H. K. Jassal, T. Padmannabhan, Phys. Rev. D 67063504 (2003).

11. M. C. Bento, O. Bertolami, A. A. Sen, Phys. Rev. D 66 043507 (2002);

D. J. Liu, X. Z. Li, Chin. Phys. Lett.22 1600 (2005)

12. G. 't Hooft, Dimensional reduction in quantum gravity, [arXiv: gr-qc/9310026;

L. Susskind, J. math. phys. 366377 (1995) [arXiv: hep-th/9409089v2]; W. Fischler and L. Susskind, Holography and cosmology, arXiv:hep-th/9806039;

E. Witten, Adv. Theor.Math. Phys. 2 253(1998).

13. A. Cohen, D. Kaplan and A. Nelson, Phys. Rev. Lett. 82 4971 (1999).

14. F. Károlyházy, Nuovo Cimento, A 42390 (1966); Naoki Sasakura, Prog.Theor. Phys. 102 169(1999) [arXiv: hep-th/9903146];

M. Maziashvili, Int. J. Mod. Phys. D 16 1531(2007) [arXiv: gr-qc/0612110];

M. Maziashvili, Phys. Lett. B 652165 (2007)[arXiv: 0705.0924].

15. R. -G. Cai, Phys. Lett. B 657228 (2007) [arXiv: 0707.4049];

H. Wei, R. -G. Cai, Phys. Lett. B 660113 (2008)[arXiv: 0708.0884].

16. H. Wei, R. -G. Cai, Eur. Phys. J. C 5999 (2009) [arXiv: 0707.4052].

17. A. Sheykhi, Phys. Lett. B 680113 (2009) [arXiv: 0907.5144].

18. O. A. Lemets, D. A. Yerokhin, L. G. Zazunov, JCAP 01, 007 (2011) arXiv:1010.0567.

19. V. Faraoni, Cosmology in Scalar-Tensor Gravity, Kulwer, Boston, 2004;

E. Elizalde, S. Nojiri, S. D. Odintsov, P. Wang, Phys. Rev. D 71103504 (2005);

S. Nojiri, S. D. Odintsov, Gen. Rel. Grav. 381285 (2006); R. Gannouji, et al., JCAP 0609016 (2006).

20. C. Brans, R. H. Dicke, Phys. Rev. 124925 (1961).

21. Y. G. Gong, Phys. Rev. D 70 064029(2004).

22. Y. G. Gong, J. Liu, JCAP 09 010(2008).

23. A. Sheykhi, Phys. Lett. B $681205(2009)$.

24. J. Liu, Y. G. Gong and X. M. Chen, Phys. Rev. D 81 $083536(2010)$

25. K. Xiao, and J. Y. Zhu, Int. J. Mod. Phys. A26 4993(2010).

26. L. Amendola, Phys. Rev. D 62 043511(2000); L. Amendola, C. Quercellini, Phys. Rev.D 68 023514(2003);

L. Amendola, S. Tsujikawa, M. Sami, Phys. Lett. B 632 155(2006).

27. D. Pavon, W. Zimdahl, Phys. Lett. B 628206 (2005); S. Campo, R. Herrera, D. Pavon, Phys. Rev. D 78 021302(R) (2008). 
28. C. G. Boehmer, G. Caldera-Cabral, R. Lazkoz, R. Maartens, Phys. Rev. D 78023505 (2008).

29. S. B. Chen, B. Wang, J. L. Jing, Phys. Rev. D 78123503 (2008).

30. R. Jimenez, L. Verde, T. Treu and D. Stern, Astrophys. J. 593622 (2003).

31. J. Simon, L. Verde and R. Jimenez, Phys. Rev. D 71 123001(2005).

32. D. Stern, R. Jimenez, L. Verde, M. Kamionkowski and S. A. Stanford, JCAP 02 08(2010).

33. C. Ma, T. J. Zhang, Astrophys. J. 73074 (2011) .

34. Z. X. Zhai, T. J. Zhang, W. B. Liu. JCAP 08019 (2011).

35. M. Arik, M. Calik, M. B. Sheftel, Int. J. Mod. Phys. D $17225(2008)$

36. L.P.Chimento,Phys.Rev.D 81043525 (2010).

37. W.Zimdahl, D.Pavon, L. P. Chimento, Phys.Lett. B 521 133 (2001).

38. H. Wei, R. -G. Cai, Phys. Rev. D 71 043504(2005) hep-th/0412045.

39. Z. -K. Guo, R. -G. Cai, Y. -Z. Zhang, JCAP $05002(2005)$ astro-ph/0412624.

40. Y. H. Li, X. Zhang, Eur. Phys. J. C 711700 (2011)

41. N. Banerjee, D. Pavon, Phys. Lett. B 647477 (2007).

42. Yungui Gong, Phys. Rev. D 61043505 (2000) arxiv:gr-qc/9909013; On holography in BransDicke cosmology, arXiv:gr-qc/9911086]. 